\title{
A General Measure of Human Population Growth Regulation ${ }^{1}$
}

\author{
KENNETH M. WEISS \\ Department of Human Genetics, University of Michigan Medical School, \\ Ann Arbor, Michigan 48104
}

\begin{abstract}
KEY WORDS Population regulation - Population growth Fertility - Mortality · Population evolution.
\end{abstract}

\begin{abstract}
A general measure relating the relative effects of mortality and fertility in damping population growth has been derived from stable population theory. This measure, called the Index of Growth Regulation, can be calculated from a life table and a fertility schedule. It is a single number which integrates the fertility and mortality aspects of a population. It has intuitive meaning, and can be related to social aspects of the population. It can be used to compare any two populations, and for this purpose it has advantages over traditional comparative statistics such as life expectancy, which consider only mortality.

By selecting life tables representing general stages of human cultural evolution, it has been shown that hunting-gathering populations were regulated more by mortality than by fertility factors. That is, more growth which could have occurred did not occur due to the incidence of mortality than to the incidence of infecundity. The two forces were of about equal weight before the industrial revolution, and fertility has had a predominant role in population regulation since the beginning of industrialization.
\end{abstract}

JUAN COMAS AWARD-1972

Many contemporary scientists are concerned with analyzing the processes by which human populations regulate their growth. This concern derives from several separate interests, including genetic, ecological, and purely demographic ones. This paper will examine some basic elements of population regulation and will introduce new measures for this which are of special interest and relevance to anthropologists.

The only two major biological factors which are ultimately involved with the regulation of population growth are fertility and mortality. The relative effects of these two forces in a given population have been examined in several ways. From a genetic point of view Crow ('58) introduced his Index of Total Selection, now often called the Index of the Opportunity for Selection. This index partitions variance in mean fitness into a component associated with differential fertility within the population and one associated with differential mortality. This approach reveals, for a given case, the relative importance of these two forces for natural selection. In this way, Crow relates Fisher's Fundamental Theorem of Natural Selection to the demographic structure of a population (see Cavalli-Sforza and Bodmer, '71).

Other studies have related the growth potential of a population to its fertility and mortality structure by examining the effects of changes in that structure on growth (Cole, '54; Lewontin, '65; Meats, '71). Recently many articles have appeared which relate growth rates, demographic structure (the results of mortality and fertility patterns) and genetic considerations; among these are Charlesworth (70), Charlesworth and Giesel (72a,b), Anderson and King ('70) and King and Anderson (71). There is, therefore, a growing con-

1 A short version of this paper was read at the 1972 meetings of the American Association of Physical Anthropologists. 
cern among zoologists with the relations between growth rates, fertility and mortality structure, and genetics.

From a more purely demographic standpoint, Coale (57) showed that for a given population the effects of changes in the fertility schedule are far greater than are those of changes in the mortality schedule on the stable age distribution which results. This did not relate to growth rates, and if they are held constant the relative effects of fertility and mortality changes would differ (and be more difficult to ascertain) from those obtained by Coale.

For much that is of interest to anthropologists studying the effects of biological and cultural evolution on human populations, one would like to be able to relate the fertility and mortality schedules to each other as they affect the population while at the same time maintaining some control over what happens to the growth rate. The value of this kind of measure will become clear.

In general, when one talks of population regulation one is referring to the damping or curbing of growth. That is, most animal populations exhibit approximately zero growth, whereas they are physiologically capable of rapid growth. Much of the "new" biology with its emphasis on the behavior of natural populations concerns the social-behavioral means by which populations limit their fertility. Selection for self-regulating behavior has doubtlessly occurred in higher vertebrates, although whether by the means advanced by Wynne-Edwards ('62) or not (Williams, '66) is open to question. A discussion of various aspects of the question is in McLaren ('71). For the present, the important point is that the spacing, growth, and fertility regulation are closely connected through animal behavior. The case is similar for anthropologists, in that much of interest to us concerns the way in which populations are spaced and their growth controlled, in particular the way fertility is regulated.

The result of much of the work done on animal behavior conflicts with the Darwinian view of natural selection. That view, that selection generally works by weeding the less fit from an overproduced offspring cohort, is now considered an oversimplification. Nature does not gen- erally operate by crises pléthoriques, as it were, but by preventing the birth of excess offspring, in many higher vertebrates. Mortality of course also plays a part in population regulation, and hence it is important to be able to relate the relative effects of both fertility and mortality in the non-growth of an animal population.

In the case of humans it is not unreasonable to assume that our ancestral populations were generally non-growing ones. This was due to myriad "natural" forces of mortality (accident, disease, etc.) and some fertility controls of a "natural" kind (e.g., nutritional deficiencies affecting fecundity). In addition, however, there are many cultural factors by which we affect both our mortality and fertility. Among these are warfare, murder, infanticide, abortion, contraception, bride price and delayed marriage, infant neglect, lactation intercourse taboos, and so on. What are the net effects of these combined factors in our population control? How much growth would occur in their absence?

\section{Population theory}

One can measure the relative effects of fertility and mortality in population control by appealing to stable population theory and extracting the required measures. The general theory of stable populations is given in most detailed exposition in Keyfitz ('68) to which the reader is referred. A population's age structure, growth, birth, and death rates are all determined by the age-specific mortality and fertility rates which exist. If these rates are fixed, and if there is no net migration to or from the population, the age structure of the population (the per cent of the group at each age) will become fixed. This is the stable age distribution, so called because if the mortality and fertility schedules are temporarily perturbed and then returned to their orignal values the age distribution also will return to the stable form. This age distribution is completely defined by the following equations:

$$
\begin{aligned}
& 1=\int_{0}^{\infty} e^{-r x} p(x) f(x) d x \\
& 1=\int_{0}^{\infty} b e^{-r x} p(x) d x
\end{aligned}
$$


where $p(x)$ is the chance of surviving from birth to age $x, f(x)$ is the chance a female age $x$ will give birth to another female child, $b$ is the overall (crude) birthrate per person per year, and $r$ is the growth rate per year. $r$ equals the crude birth rate minus the crude death rate; if these rates are equal, $r=0$, and the population is called stationary, which is a special case of the stable population.

The important part of equation (1) for us is the function $\emptyset(x)=p(x) f(x)$, called the Net Maternity Function. Its sum over all fertile ages (generally 15 to 50 in human populations) is equal to the number of times each female born will, on average, replace herself before she dies. It therefore represents the behavior of an entire cohort or generation of individuals. This sum, called the Net Reproduction Rate (NRR) and abbreviated $R_{o}$ in most demographic literature, is equal to 1.0 in a stationary (non-growing) population, and is greater than 1.0 if the population is growing since each female will more than replace herself in such a case.

If $R_{0}$ exceeds 1.0 and the population is therefore growing, we can determine the annual growth rate from its value $\left(R_{o}\right.$ measures the growth proportion for an entire generation). This is given by the relation

$$
R_{o}=e^{r^{T}}
$$

where $\mathrm{T}$ is the generation length, which is approximately equal to the average age of childbearing.

Whenever reproduction does not occur which under some "ideal" (maximal) conditions might occur, we will say that the population's growth is thereby damped. When this is due to the death of a potential breeder who under the ideal circumstances would have bred, we will define this as mortality damping; when it is due to the failure to breed of a living individual who under the ideal conditions would have bred, we define this as fertility damping. These terms are to be applied regardless of the specific reason for the failure to reproduce (that is, regardless of the specific cause of mortality or infecundity). If we can specify the ideal rates of mortality and fertility, namely those which would produce maximum growth, then we can compare any actual population against the ideal and determine how much of the growth differential between actual and ideal is due to fertility damping and how much to mortality damping.

The amount of mortality damping is defined as the difference between the growth rate actually observed, $r$, and that which would result from ideal mortality conditions and the observed fertility, call it $r_{m}$. The survivorship function, $p(x)$, is equivalent to specifying mortality rates. An ideal mortality schedule could be approximated by assuming that in human populations the only individuals not physiologically capable of living at least to age 50 (through their reproductive span) were those with genetic defects. All others could be said not to be fated to an early death. Since such genetic defects are very rare, it is not unreasonable to approximate ideal mortality conditions by saying that $p(x)=1.0$ up through age 50. In fact, in modern societies $p(50)$ can be up to or greater than 0.95 , as is the case for Norway recently (Keyfitz and Flieger, '68). Thus, complete survivorship through all of reproductive years is not an unrealistic "ideal." In such a case, the Net Maternity Function would be

(4)

$$
\emptyset_{m}(x)=1.0 f(x) \text {, }
$$

and mortality damping is

(5) $\quad G_{m}=r_{m}-r$.

Similarly for fertility, we can estimate the amount of fertility damping by comparing an actual growth rate with that which would occur with maximized fertility and the observed mortality. This requires the determination of an ideal fertility schedule, $f^{\prime}(x)$, with which the Net Maternity Function would be

(6) $\quad \emptyset_{f}(x)=p(x) f^{\prime}(x)$,

and the fertility damping is

(7) $\quad G_{f}=r_{f}-r$.

Given the values of mortality damping, $G_{m}$, and fertility damping, $G_{f}$, we can define the total Index of Growth Regulation (IGR) as

$$
G=\frac{G_{f}}{G_{m}} .
$$

When the population's growth, as contrasted with the ideal, is more damped by fertility factors than by mortality factors the value of $G$ will exceed 1.0, when 
the forces are equally effective $G=1.0$, and if mortality dominates $G$ will be less than 1.0 .

The Index of Growth Regulation is a single number assigned to a given population which relates the fertility and mortality patterns of that population to each other. It is a number by which any two populations may be compared, and it has intuitive meaning. Most other statistics now used to compare life tables are related to crude birth or death rates or to life expectancy; hence they do not integrate aspects of the population structure as the IGR does. For the uses to which it can be put in anthropology, the IGR has many advantages over these other statistics.

The computation of the IGR requires the determination of three growth rates: $r$ derived from $\emptyset(\mathbf{x}), r_{m}$ derived from $\emptyset_{m}(x)$, and $r_{f}$ derived from $\emptyset_{f}(x)$. Compucing growth rates is not a direct procedure, since $r$ is the real root of equation (1) which is only implicitly given by that equation. There are many methods for solving for $r$; that used in this paper involves computing various cumulants of the probability density function $\emptyset(x) / R_{o}$, and using iteration methods for approximating $r$. These calculations are easily made using a computer; their details can be found in Keyfitz ('68) chapter 5 and chapter 6 section 2 .

The basic computations discussed thus far can be made from abridged life tables, using approximations, for the integrals, which are summations over age classes; see Barclay (58) for a discussion of the life table.

For a simpler means of estimating the same demographic status of a population from its life table, one can deal with $\emptyset(x)$ values directly without having to solve for growth rates. By summing $\emptyset(x)$, $\emptyset_{m}(x)$, and $\emptyset_{f}(x)$ over all fertile years, one derives the actual NRR $\left(R_{0}\right)$, a similar value for ideal mortality and observed fertility $\left(R_{\eta 1}\right)$ and one for observed mortality and ideal fertility $\left(R_{f}\right)$, respectively. These values do not represent annual growth rates as the values in equations (5) and (7) do, but rather the behavior of a cohort of individuals over their entire lifetime. The Net Reproduction Rate is in units of cohort replacements per co- hort, and represents the number of times a cohort of individuals replaces itself in one generation. To compute a " $G$ " value from NRR's rather than $r$ 's is to look at generation behavior rather than annual results. To be informed of the growth "rate" of a generation of individuals is, however, not equivalent to knowing the annual growth rate of a population made up of those individuals. This is because, although there is a unique relationship between $R_{o}$ and $r$ (given by equation (3)), this only obtains for a fixed generation length, $T$. The generation length is determined by the parameters in $\emptyset(x)$ and by the growth rate (see Keyfitz, '68), and hence it is possible for two populations to have the same $R_{o}$ but different $r$ values. The difference in generation lengths for life tables from reasonably similar populations is slight, however, and the simpler method of approximating $G$ is about as informative as the more "elegant" formulation in terms of growth rates.

\section{Application of the Index of Growth Regulation}

We can now turn to an important and interesting question regarding the evolution of human cultures: has cultural evolution changed in a systematic way the relative importance of fertility and mortality factors in the damping of population growth? This question can be answered in a quantitative way by the IGR.

There are many ways of viewing the changes which have probably occurred in the course of human cultural evolution. Most anthropologists would probably subscribe to some sort of series of cultural stages through which the majority of human cultures in some sense can be said to have passed. White (59) and Service ('62) discuss a rather general and acceptable schema for this; while many would argue with the details of the social characteristics applicable to various stages, and others object to the use of "stages" at all to represent a continuum of variety and change, surely some general things can be said about cultural evolution. We can agree that most of human history has been lived in hunting-gathering bands; following this there gradually arose swidden agricultural groups (or groups with 
some similar quasi-fixed settlement ecology); from such groups the development of urbanized agricultural states occurred. These persisted for a long portion of recorded history until the industrial revolution wrought dramatic changes in the entire human life style.

Because the available data from anthropological sources allow little discrimination beyond such a broad schema, and because such broad categories are general enough that they need not be regarded as rigid types, it is not unreasonable to group human cultures of pre-industrial times as hunter-gatherer, proto-agriculture, and urban agriculture. It is possible, in fact, to represent common demographic conditions among populations at these levels, since many sets of data from similar cultures show similar demographic characteristics. This has been done by the author elsewhere (Weiss, '72a). Life tables were taken from reported survivorship data, and a fertility schedule was computed based on an assumed zerogrowth condition for the various cultural stages by a method in Weiss ('72a,b).

The assumption of zero growth is consistent with most feeling about human ecology; even urban agricultural states have until recently been growing at rates very close to zero, and of course this is the only equilibrium condition for any longterm biological population.

From life tables (with fertility schedules) representing these stages of preindustrial cultures and a series of tables representing nations at various times in the industrial revolution, values of the IGR can be computed. This has been done, and the results are shown in table 1 . The data from Guatemala 1893 are taken from Arriaga ('68) and are included to represent a tropical "underdeveloped" colonial nation with high mortality; such populations are common now and its seems probable that a large proportion of human beings who have ever lived have lived in such populations. There were no fertility data, and so a zero-growth fertility schedule was computed for Guatemala. The other recent data are from Keyfitz and Flieger (68).

The values of $G_{f}$ and $G_{m}$ represent growth rate differentials between the actual growth rate, $r$, and that which would occur under ideal fertility and ideal mortality, respectively. The calculation of $G_{f}$ requires an ideal or maximal fertility schedule. The basic method was that given in Weiss $(72 a, b)$ with the assumption that a female, at the height of her fecundity, could produce no more than one offspring every 18 months, on average. This maximum value was used throughout the calculations, and since modern cultures, with soft foods and consequent early weaning, can probably reproduce faster than this the assumption will reduce the value of $G_{f}$ for recent cultures. This will increase the significance of the pattern shown in table 1.

The IGR values for cultures at various "points" along a cultural evolutionary schema show a clear and consistent trend. Fertility damping has become steadily more important in the control of population growth, relative to mortality. In hunting-gathering cultures, fertility was only about $30.8 \%$ as important as mortality, whereas in most recent times it is about 55.8 times as important. Fertility has

TABLE 1

Growth regulation index and components for selected populations

\begin{tabular}{lcccc}
\hline \multicolumn{1}{c}{ Population } & $G_{\boldsymbol{f}}$ & $\boldsymbol{G}_{\boldsymbol{m}}$ & $\boldsymbol{G}$ & $\boldsymbol{r}$ \\
\hline Hunter-gatherers & 0.0162 & 0.0527 & $\boldsymbol{0 . 3 0 8}$ & 0.000 \\
Proto-agriculture & 0.0235 & 0.0451 & $\mathbf{0 . 5 2 0}$ & 0.000 \\
Urban agriculture & 0.0281 & 0.0402 & $\mathbf{0 . 6 9 7}$ & 0.000 \\
Guatemala 1893 & 0.0332 & 0.0346 & $\mathbf{0 . 9 5 9}$ & 0.000 \\
Sweden 1780 & 0.0418 & 0.0188 & $\mathbf{2 . 2 2 2}$ & 0.006 \\
U. K. 1861 & 0.0416 & $\mathbf{0 . 0 1 5 7}$ & $\mathbf{2 . 6 5 1}$ & 0.010 \\
Sweden 1903 & 0.0487 & $\mathbf{0 . 0 0 8 2}$ & $\mathbf{5 . 9 4 0}$ & 0.011 \\
Norway 1964 & 0.0558 & 0.0010 & $\mathbf{5 5 . 8 0 0}$ & 0.012 \\
\hline
\end{tabular}

1 Actual fertility data unavailable; based on fertility-adjusted zero-growth model. 
gained in relative importance apace with industrialization and population growth. Just prior to industrialization the two factors were of about equal significance. Of course since these values come from life tables representing an average of a wide range of experience, they are only approximate; but the trend is clear and irrefutable.

The reason for the acceleration in the relative importance of fertility during the industrial revolution is that mortality declined dramatically. This cannot easily be explained, since medical advances were very slow in becoming effective in reducing mortality rates (McKeown and Brown, '55; McKeown and Record, '62). Economic improvements probably resulted in better living conditions generally, and hence better nutrition and sanitation in urban settings (Thompson, '53). It seems that European fertility rates changed relatively little during most of this period, and the "demographic transition" which occurred there is unique to human history. An interesting comparison of this with what occurs in "underdeveloped" nations is given by Arriaga (70), who compares the European phenomenon with that in Latin America this century. "Underdeveloped" nations probably cannot undergo the same kind of transition experienced in Europe; for this reason the Guatemala data represent a condition not comparable with any longterm condition of Europe.

Since the interesting aspects of the changes revealed in table 1 center around the human behavior involved, a discussion of the meaning of the values of $G$ raises an interesting question. Should infanticide be considered a behavior related to growth damping by morality or by fertility? Surely the behavioral distinction between abortion and infanticide is a tenuous one, and abortion is always considered among the means of controlling fertility. Infanticide has probably always been an important factor in human demography; it is known widely in pre-industrial societies, and has even been surprisingly important in the European tradition (Langer, '72).

In the computation of $G$ in table 1 , infanticide is considered as a mortality factor; that is, all live births are considered to have entered the population, and their deaths are a component of the infant mortality rate. This is partly a pragmatic decision, since most anthropological data do not allow the partitioning of infant mortality by cause. It is given support, in addition, by the fact that many infants who are killed by their parents in preindustrial societies would very likely have died anyway. They are selected for infanticide in many cases because they are weak or deformed, because their mother is too young to nurse them successfully, because the environment cannot support them, or because they have siblings who are still dependent on nursing (Krzywicki, '34; Carr-Saunders, '22). These infant deaths, at least, should be considered as part of the incidence of mortality, and the remainder of infanticides would make little difference to the value of $G$.

If an industrialized nation were to become a stable, equilibrium cultural stage it would have to do so by reducing growth to zero. This is unlikely to occur by a voluntary increase in mortality rates; it is more likely that, as is occurring in many nations today, fertility rates will be lowered through various behavioral means. In fact, if Norway 1964 were to reduce its fertility to a zero-growth level its value of $G$ would become 67.8 , a noticeable change from its actual value of 55.8. It appears that, if medical advances are not countered with new sources of mortality which result from high population densities, the potential effects of mortality will diminish steadily as survivorship continues to increase. Eventually, the relative effect of fertility will rise without limit as it becomes the only demographic factor involved with the potential alteration of population growth.

\section{LITERATURE CITED}

Anderson, W. W., and C. E. King 1970 Agespecific selection. Proc., Nat. Acad. Sci., 66: $780-786$.

Arriaga, E. E. 1968 New Life Tables for Latin American Populations. Population Monograph Series, 3, University of California Institute of International Studies, Berkeley.

1970 Mortality Decline and Its Demographic Effects in Latin America. Population Monograph Series, 6, University of California Institute of International Studies, Berkeley.

Barclay, G. W. 1958 Techniques of Population Analysis. John Wiley, New York.

Carr-Saunders, A. M. 1922 The Population Problem: a Study in Human Evolution. Clarendon Press, Oxford. 
Cavalli-Sforza, L. L, and W. F. Bodmer 1971 The Genetics of Human Populations. W. H. Freeman, San Francisco.

Charlesworth, B. 1970 Selection in populations with overlapping generations. I. The use of Malthusian parameters in population genetics. Theor. Pop. Biol., 1 : 352-370.

Charlesworth, B., and J. T. Giesel 1972a Selection in populations with overlapping generations. II. Relations between gene frequency and demographic variables. Am. Nat., 106: 388-401.

$1972 \mathrm{~b}$ Selection in populations with overlapping generations. IV. Fluctuations in gene frequency with density-dependent selection. Am. Nat., 106: 402-411.

Coale, A. J. 1957 How the age distribution of a human population is determined. Cold Spring Harbor Symposia on Quantitative Biology, XXII: 83-90.

Cole, L. C. 1954 The population consequences of life history phenomena. Quart. Rev. Biol., 29: 103-137.

Crow, J. F. 1958 Some possibilities for measuring selection intensities in man. Human Biol., 30: 1-13.

Keyfitz, N. 1968 Introduction to the Mathematics of Population. Addison-Wesley, Reading, Mass.

Keyfitz, N., and W. Flieger 1968 World Population: an Analysis of Vital Data. University of Chicago Press, Chicago.

King, C. E., and W. W. Anderson 1971 Agespecific selection. II. The interaction between $r$ and $K$ during population growth. Am. Nat., 105: 137-156.

Krzywicki, K. 1934 Primitive Society and Its Vital Statistics. Macmillan, London.
Langer, W. L. 1972 Checks on population growth: 1750-1850. Sci. Am., 226: 92-99.

Lewontin, R. C. 1965 Selection for colonizing ability. In: The Genetics of Colonizing Species. G. Baker and L. Stebbins, eds. Academic Press, New York, pp. 79-94.

Mckeown, T., and R. G. Brown 1955 Medical evidence related to English population changes in the eighteenth century. Pop, Studies, IX: 119-141.

Mckeown, T., and R. G. Record 1962 Reasons for the decline of mortality in England and Wales during the nineteenth century. Pop. Studies, XVI: 94-122.

McLaren, I. A. (ed.) 1971 Natural Regulation of Animal Populations. Atherton, New York.

Meats, A. 1971 The relative importance to population increase of fluctuations in mortality, fecundity and the time variables of the reproductive schedule. Oecologia, 6: 223-237.

Service, E. R. 1962 Primitive Social Organization. Random House, New York.

Thompson, W. S. 1953 Population Problems. Fourth ed. McGraw-Hill, New York.

Weiss, K. M. 1972a Model Life Tables for Preindustrial Populations. Ph.D. thesis, University of Michigan.

$1972 \mathrm{~b}$ A method for approximating agespecific fertility in the construction of life tables for earlier human populations. In preparation.

White, L. A. 1959 The Evolution of Culture: the Development of Civilization to the Fall of Rome. McGraw-Hill, New York.

Williams, G. C. 1966 Adaptation and Natural Selection. Princeton University Press, Princeton.

Wynne-Edwards, V. C. 1962 Animal Dispersion in Relation to Social Behaviour. Hafner, New York. 